\title{
Delayed Immunosuppressive Treatment in Life-Threatening Paraquat Ingestion: A Case Report
}

\author{
Alexis Descatha, $M D^{a, b}$, Bruno Mégarbane, $M D, P h D^{a}$, Véronique Garcia, $M D^{c}$, \\ Frédéric J. Baud, $\mathrm{MD}^{a}$
}

aAssistance Publique-Hôpitaux de Paris, Hôpital Lariboisière, Réanimation Médicale et Toxicologique, Paris, France

bAssistance Publique-Hôpitaux de Paris, Hôpital Raymond Poincaré, Service de Médecine du Travail, Garches, France

cCentre Hospitalo-Universitaire Maison Blanche, Service de Pneumologie, Reims, France

\begin{abstract}
Introduction: Combined glucocorticoids and cyclophosphamide pulse therapy showed promising results in moderate-to-severe paraquat poisonings to reduce life-threatening respiratory complications. Its benefit has been observed when given early in the course of poisoning; however, whether its delayed administration remains beneficial is unknown.

Case Report: We describe a 23-year-old male who ingested $70 \mathrm{~mL}$ of a commercialized concentrate formulation with $20 \%$ weight/volume paraquat in a suicide attempt. Within 24 hours from paraquat ingestion, he presented most of the indicators of poor outcome, including gastritis, early renal dysfunction, dark blue urine colorimetric dithionite test, and marked plasma paraquat concentrations $(0.56 \mu \mathrm{g} / \mathrm{mL}$ at 13 hours, and $0.41 \mu \mathrm{g} / \mathrm{mL}$ at 24 hours after ingestion). The patient received early gastrointestinal decontamination and aggressive supportive treatments. However, due to a rapidly progressive severe pulmonary infection, glucocorticoids and cyclophosphamide were delayed until day 14. Interestingly, our patient survived with mild respiratory sequelae despite poor initial prognosis.

Discussion: This observation suggests the potential benefit of immunosuppressive pulse therapy, even if administered 14 days after paraquat ingestion, and highlights the role of paraquat-induced alveolitis in the development of fibrosis.

Conclusion: Combined glucocorticoids and cyclophosphamide should be considered in moderate-to-severe paraquat poisonings, even if delayed.
\end{abstract}

\section{INTRODUCTION}

Paraquat, or 1,1-dimethyl-4,4-bipyridilium dichloride, is a widelyused herbicide. Moderate-to-severe poisonings may result in acute renal failure, hepatitis, and diffuse alveolitis followed by extensive pulmonary fibrosis. Death resulting from hypoxemia secondary to lung fibrosis usually occurs within 2-3 weeks [1]. In a subgroup of patients who are yet to be defined, case reports and prospective studies have suggested the possible interest of pulse therapy (i.e., high-dose intermittent intravenous infusions) of immunosuppressive treatments. In these studies, cyclophosphamide $(15 \mathrm{mg} / \mathrm{kg}$ per day for 2 days) administered simultaneously with glucocorticoids (methylprednisolone $1 \mathrm{~g} /$ day for 3 days, followed by dexamethasone $30 \mathrm{mg}$ /day for 14 days) was initiated within 24-48 hours after ingestion [2-6]. Other possibly effective therapies have been suggested, such as oral adsorbents (to decrease paraquat absorp-

Keywords: paraquat, acute poisoning, respiratory failure, prognosis, pulse therapy

Acknowledgment: The authors would like to acknowledge Alexandra Chronopoulos, MD, FRCPC, Intensive Care and Internal Medicine, Hôtel-Dieu de Lévis, Québec, Canada, for her helpful review of this manuscript.

Notes: There was no outside funding of any kind used for this study.

Corresponding author: Bruno Mégarbane, Réanimation Médicale et Toxicologique, Hôpital Lariboisière, 2 rue Ambroise Paré, 75010 Paris, France. E-mail: bruno-megarbane@wanadoo.fr 
tion), hemodialysis or hemoperfusion (to enhance paraquat elimination), antioxidant agents (to prevent and scavenge reactive oxygen species or repair induced lesions), and lung transplant [1]. However, their efficacy is questionable and has never been proved in any controlled study. Here we report the case of a paraquatpoisoned patient who survived with limited respiratory dysfunction despite initial indicators of poor outcome. This observation suggests that our patient's good outcome was associated with aggressive supportive treatments and delayed administration of glucocorticoids and cyclophosphamide.

\section{CASE REPORT}

A 23-year-old vineyard worker with no significant past medical history ingested $70 \mathrm{~mL}$ of Gramoxone solution (20\% weight/ volume paraquat) in a suicide attempt. Because of vomiting and abdominal pain 1 hour following paraquat ingestion, he presented himself to a county hospital. Gastric lavage was immediately initiated. Activated charcoal $(50 \mathrm{~g})$ and $\mathrm{N}$-acetylcysteine infusion (300 mg/kg per day) were administered within 2 hours of the ingestion. The patient was then referred to our toxicological intensive care unit (ICU) 12 hours after ingestion. On admission, he was febrile $\left(38.5^{\circ} \mathrm{C}\right)$ and presented normal vital signs (blood pressure, 130/75 $\mathrm{mmHg}$; pulse rate, 90/min; respiratory rate, 18/min; SpO2, 97\%; Glasgow coma score, 15). Physical examination was normal, except for painful erosions in the oral cavity. Laboratory tests showed an elevation of serum creatinine concentration (144 $\mu \mathrm{mol} / \mathrm{L} ; \mathrm{N}, 50-105)$ and white blood cell count $\left(18.10^{9} / \mathrm{L} ; \mathrm{N}, 4-10\right)$. Analysis of arterial blood gases while breathing room air showed the following: $\mathrm{pH} 7.47$ (N, 7.34-7.35), $\mathrm{PaO} 2,95 \mathrm{mmHg}(\mathrm{N}, 75-100), \mathrm{PaCO} 2,33 \mathrm{mmHg}(\mathrm{N}, 32-45)$, and plasma bicarbonate concentration $24.0 \mathrm{mmol} / \mathrm{L}(\mathrm{N}, 20.0-26.0)$. Gastroscopy revealed esophagitis and erythematous fundic gastritis. Chest radiographs showed a limited left basal alveolar consolidation. Intravenous fluids and amoxicillin plus clavulanic acid (3 g/day) were administered.

The initial urine colorimetric sodium dithionite test showed dark blue coloration. This test is based on the reduction of paraquat by sodium thionite under alkaline conditions to form its stable blue radical ion. Navy blue or dark blue colorations indicate significant paraquat ingestion, while light blue coloration or the absence of color indicates mild poisoning. Serum paraquat concentrations, measured using high pressure liquid chromatography, were: $1.75 \mu \mathrm{g} / \mathrm{ml}$ at 4 hours, $0.56 \mu \mathrm{g} / \mathrm{mL}$ at 13 hours, and 0.41 $\mu \mathrm{g} / \mathrm{mL}$ at 24 hours after paraquat ingestion.

Rapidly after ICU admission, respiratory symptoms developed. Dyspnea gradually worsened despite early antibiotics. On day 5, the patient developed respiratory failure with a breathing rate of $50 / \mathrm{min}$ and a $\mathrm{PaO} 2 / \mathrm{FiO} 2$ ratio of $243 \mathrm{mmHg}(\mathrm{N}>350)$. Noninvasive ventilation with positive end-expiratory pressure under air and nitric oxide (NO) was started, allowing an increase in $\mathrm{PaO} 2 / \mathrm{FiO} 2$ ratio $(470 \mathrm{mmHg}$ ). On day 9 , the patient became suddenly agitated, his cardiovascular condition deteriorated (blood pressure, 85/30 mmHg; pulse rate, 130/min), and his $\mathrm{PaO} 2 / \mathrm{FiO} 2$ ratio worsened $(124 \mathrm{mmHg})$, subsequently requiring intubation and norepinephrine $(0.8 \mu \mathrm{g} / \mathrm{kg}$ per minute) support. Chest computed tomography (CT) scans showed extensive bilateral pulmonary infiltrates, consistent with acute lung injury (Figure 1A). Given his history, clinical presentation, and $\mathrm{PaO} 2 / \mathrm{FiO} 2$ ratio, the patient met the definition of acute respiratory distress syndrome [7]. A Pseudomonas aeruginosa hospital-acquired pneumonia, empyema, and bacteremia were diagnosed and successfully treated with drainage, ciprofloxacine, and ticarcillin.

Four days later, despite fever resolution, reduction in respiratory secretions, vasopressor weaning, and negative blood and pulmonary cultures, all suggestive of severe sepsis resolution, there was no significant improvement in the patient's respiratory function. Thus, due to the severity of the patient's condition as well as to a suspected direct role of paraquat in lung injury, the decision to administer immunosuppressive therapy was undertaken at day 14 (322 hours following paraquat ingestion). The protocol included $1 \mathrm{~g}$ methylprednisolone in 100-mL D5W over 1 hour daily for 3 days, $15 \mathrm{mg} / \mathrm{kg}$ cyclophosphamide in 100-mL D5W over 1 hour daily for 2 days, and $10 \mathrm{mg}$ dexamethasone every 8 hours daily for 14 days. $\mathrm{N}$-acetylcysteine infusion was discontinued on day 28. A tracheostomy was performed on day 30 and

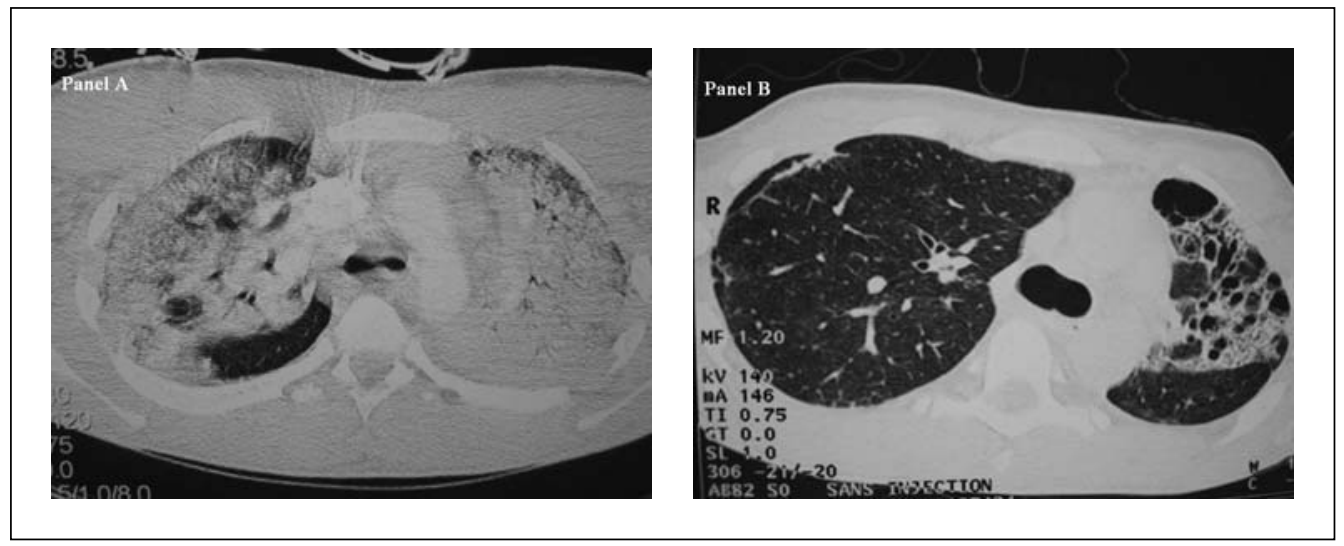

Figure 1: Evolution of chest CT scans, showing bilateral alveolar and interstitial opacities on day 9 (A) and left bronchiectasis, with bullous paraseptal atelectasis and fibrosis on day 72 (B). 
spontaneous ventilation became possible on day 51. ICU-acquired paresis progressively recovered, with residual limb muscle atrophy. The patient was discharged from the ICU on day 72 with residual effort dyspnea. Arterial blood gases at rest on room air were as follows: $\mathrm{pH} 7.43, \mathrm{PaO} 275 \mathrm{mmHg}, \mathrm{PaCO} 246 \mathrm{mmHg}$, and plasma bicarbonate concentration $29.0 \mathrm{mmol} / \mathrm{L}$. Chest CT scans revealed residual bullous sequelae, predominating in the left lung (Figure 1B). Four months after self-poisoning, the patient had no more dyspnea with a forced expiratory volume at $1 \mathrm{~s}$ of 2270 $\mathrm{mL} /$ minute (55\% of predicted values), and pulmonary total capacity of $3830 \mathrm{~mL}$ (59\%). Chest CT scans were stable. His muscle atrophy improved. One year later, he was employed as a warehouseman and was lost to follow-up.

\section{DISCUSSION}

Paraquat intoxication is associated with a high mortality rate, resulting from either multi-organ failure (rapid death in fulminant poisonings) or progressive pulmonary damage (delayed death in moderate-to-severe poisonings). Our paraquat-poisoned patient presented most of the validated indicators of poor outcome, including suicidal ingestion, ingestion of a large quantity (one mouthful of a $20 \%$ solution is potentially life-threatening), the presence of gastritis at gastroscopic examination, an early renal dysfunction, and elevated plasma paraquat concentration related to time of ingestion $[1,8]$. According to the survival probability employing Proudfoot's criteria [9] or Scherrmann's predictive value of plasma paraquat concentration [10], this patient was initially classified as likely to die. According to the Hart nomogram [11], his predicted survival rate was of $20-30 \%$. He was most likely to die from pulmonary fibrosis.

To limit paraquat-induced fibrosis, we chose noninvasive ventilation with positive end-expiratory pressure under air and nitric oxide (10 ppm), adding oxygen when $\mathrm{PaO} 2 / \mathrm{FiO} 2$ fell below $200 \mathrm{mmHg}$. Tracheal intubation was performed when agitation, altered metal status, and signs of discomfort were evident. As previously reported [12], to assure patient comfort and compliance, we used an open-mask circuit through BiPAP for NO inhalation before intubation. However, this technique did not succeed in preventing worsening of respiratory status on day 9. N-acetylcysteine was administered to our patient because of its suggested moderate protective activity in paraquat poisoning [13]. However, we believe that the role of this glutathione precursor in our patient's survival is doubtful and of no clinical significance.

Immunosuppressive treatments attenuate alveolar inflammation that leads to fibrosis [2-5]. In moderate-to-severe paraquat poisonings, the early phase of alveolar type I and type II epithelial cell destruction due to NADPH depletion and peroxidation damage is followed by a proliferative phase associating alveolitis, pulmonary edema, and inflammatory cell infiltration [1]. Widespread fibrosis occurs if damage of alveolar architecture is extensive. A recent study demonstrated that a single high-dose dexamethasone administration significantly improved the survival and the lung pathological scores of paraquat-poisoned rats, including reduction in lipid peroxidaton and carbonyl group content as well as normalization of the myeloperoxidase activities [14]. In the paraquat-intoxicated patients, administration of the immunosuppressive therapies is usually initiated as soon as possible, within $24-48 \mathrm{~h}$ after admission [2-6]. In our case, combined corticosteroids and cyclophosphamide therapy was initiated only on day 14 following ingestion, when severe sepsis was controlled. We had considered that the aspiration pneumonia and hospital-acquired infection contraindicated immunosuppressive treatment. Consequently, when sepsis improved, we decided to administer the immunosuppressive treatment, due to the lethal ingested dose of paraquat.

An important question in paraquat poisoning remains when to time interventions in relation to pathogenesis. Both paraquatmediated acute alveolitis and fibrosis may coexist and contribute to severe and fatal hypoxia. Thus, we believe that possible attenuation of paraquat-induced lung inflammation using anti-inflammatory treatments should be considered as long as irreversible fibrosis is not demonstrated. In our patient, respiratory function gradually improved to nearly normal after 4 months of followup. Our case clearly emphasizes the importance of initial alveolitis before the development of pulmonary fibrosis and the therapeutic role of immunosuppressive treatments. Due to the absence of intention-to-treat-based analysis in the previously published randomized clinical trials, the definitive efficacy of these immunosuppressive treatments is still debated [15]. When $\mathrm{PaO} 2$ falls below $60 \mathrm{mmHg}$, repeated courses of immunosuppressive therapies consistently demonstrate reduced mortality rates in severe poisonings $[3,4]$. As suggested in other cases of severe poisoning with complete recovery after combined initial pulse therapy $[4,6]$, severe lung inflammation rather than fibrosis appears to cause the lethal hypoxemia of paraquat-poisoned patients during their subacute period of intoxication. Here, early and late pulmonary infections may have worsened paraquatinduced injuries.

Patients who survive acute paraquat intoxication may develop pulmonary fibrosis with restrictive pulmonary dysfunction, yet progressively improve over time [16-18]. As did our patient, the two other reported adult survivors following severe paraquat poisoning treated with combined cyclophosphamide, methylprednisolone, and dexamethasone therapy evolved favorably. One was symptom-free at 3 months postdischarge [3] and the other recovered completely 3 months later [4]. However, in all these patients, no pulmonary function tests were performed during follow-up to exclude mild restrictive respiratory sequelae. In the 15-year-old patient treated with combined therapy for a presumed lethal dose with high serum levels of paraquat [6], CT scan of the thorax on day 7 revealed areas of pulmonary densification and ground-glass attenuation at the lung base, whereas pulmonary function tests showed alteration in the monoxide carbon diffusion. At discharge on day 22, both tests were normal.

Several points should be highlighted in our observations that may limit any definitive conclusions regarding the role of pulse 
therapy in our patient's improvement. First, early gastrointestinal decontamination performed within 2 hours following paraquat ingestion may have contributed, although its exact role cannot be definitively assessed. However, further elevated plasma paraquat concentrations as well as early renal dysfunction confirmed that a significant amount of herbicide had been absorbed rapidly despite the decontamination process. Second, although a potential benefit of the pulse therapy is suggested in this report, we cannot rule out spontaneous improvement in the event of a sublethal dose of paraquat having actually been ingested.

In conclusion, our report suggests that delayed treatment of paraquat poisoning with pulse therapy of glucocorticoids and cyclophosphamide, along with aggressive supportive measures and early gastrointestinal decontamination, was associated with our patient's good outcome. However, the optimal regimen and time of administration for these treatments remains to be determined.

The authors have no potential financial conflicts of interest to report.

\section{REFERENCES}

1. Bismuth C, Garnier R, Baud FJ, et al. Paraquat poisoning. An overview of the current status. Drug Saf 1990;5: 243-251.

2. Lin JL, Leu ML, Liu YC, et al. A prospective clinical trial of pulse therapy with glucocorticoid and cyclophosphamide in moderate to severe paraquat-poisoned patients. Am J Respir Crit Care Med 1999;159:357-360.

3. Chen GH, Lin JL, Huang YK. Combined methylprednisolone and dexamethasone therapy for paraquat poisoning. Crit Care Med 2002;30:2584-2587.

4. Lin NC, Lin JL, Lin-Tan DT, et a. Combined initial cyclophosphamide with repeated methylprednisolone pulse therapy for severe paraquat poisoning from dermal exposure. J Toxicol/ Clin Toxicol 2003;41:877-881.

5. Lin JL, Lin-Tan DT, Chen KH, et al. Repeated pulse of methylprednisolone and cyclophosphamide with continuous dexamethasone therapy for patients with severe paraquat poisoning. Crit Care Med 2006;34:368-373.
6. Dinis-Oliveira, RJ, Sarmento A, Reis P, et al. Acute paraquat poisoning: report of a survival case following intake of a potential lethal dose. Pediatr Emerg Care 2006;22:537-540.

7. Bernard GR, Artigas A, Brigham KL, et al. The AmericanEuropean Consensus Conference on ARDS. Definitions, mechanisms, relevant outcomes, and clinical trial coordination.

Am J Respir Crit Care Med 1994;149:818-824.

8. Bismuth C, Garnier R, Dally S, et al. Prognosis and treatment of paraquat poisoning: a review of 28 cases. J Toxicol/Clin Toxicol 1982;19:461-474.

9. Proudfoot AT, Stewart MS, Levitt T, et al. Paraquat poisoning: significance of plasma-paraquat concentrations. Lancet 1979;2:330-332.

10. Scherrmann JM, Houze P, Bismuth C, et al. Prognostic value of plasma and urine paraquat concentration. Hum Toxicol 1987;6:91-93.

11. Hart TB, Nevitt A, Whitehead A. A new statistical approach to the prognostic significance of plasma paraquat concentrations. Lancet 1984;2:1222-1223.

12. Eisenman A, Armali Z, Raikhlin-Eisenkraft B, et al. Nitric oxide inhalation for paraquat-induced lung injury. J Toxicol/Clin Toxicol 1998;36:575-584.

13. Shum S, Hale TW, Habersang R. Reduction of paraquat toxicity by N-acetylcysteine. Vet Hum Toxicol 1987;6:91-93.

14. Dinis-Oliveira RJ, Duarte JA, Remiao F, et al. Single high dose dexamethasone treatment decreases the pathological score and increases the survival rate of paraquat-intoxicated rats. Toxicology 2006;227:73-85.

15. Eddleston M, Wilks MF, Buckley NA. Prospects for treatment of paraquat-induced lung fibrosis with immunosuppressive drugs and the need for better prediction of outcome: a systematic review. QJM 2003;96:809-824.

16. Bismuth C, Hall AH, Baud FJ, et al. Pulmonary dysfunction in survivors of acute paraquat poisoning. Vet Hum Toxicol 1996;38:220-222.

17. Lin JL, Liu L, Leu ML. Recovery of respiratory function in survivors with paraquat intoxication. Arch Environ Health 1995;50:432-439.

18. Yamashita M, Yamashita M, Ando Y. A long-term followup of lung function in survivors of paraquat poisoning. Hum Exp Toxicol 2000;19:99-103. 